PROCEEDINGS OF THE

AMERICAN MATHEMATICAL SOCIETY

Volume 129, Number 4, Pages 983-987

S 0002-9939(00)05724-5

Article electronically published on October 10, 2000

\title{
AN ORDER CHARACTERIZATION OF COMMUTATIVITY FOR $C^{*}$-ALGEBRAS
}

\author{
WEI WU
}

(Communicated by David R. Larson)

\begin{abstract}
In this paper, we investigate the problem of when a $C^{*}$-algebra is commutative through operator-monotonic increasing functions. The principal result is that the function $e^{t}, t \in[0, \infty)$, is operator-monotonic increasing on a $C^{*}$-algebra $\mathcal{A}$ if and only if $\mathcal{A}$ is commutative. Therefore, $C^{*}$-algebra $\mathcal{A}$ is commutative if and only if $e^{x+y}=e^{x} e^{y}$ in $\mathcal{A}+\mathbf{C}$ for all positive elements $x, y$ in $\mathcal{A}$.
\end{abstract}

\section{INTRODUCTION}

Let $\mathcal{A}$ be a $C^{*}$-algebra. The sets of self-adjoint and positive elements of $\mathcal{A}$ are denoted by $\mathcal{A}_{H}$ and $\mathcal{A}_{+}$, respectively. $\mathcal{A} \dot{+} \mathbf{C}$ is the $C^{*}$-algebra obtained from $\mathcal{A}$ by adjoining an identity to $\mathcal{A}$. For $x \in \mathcal{A}$, the spectrum of $x$ in $\mathcal{A}$ is denoted by $\sigma(x)$. If $\mathcal{A}$ is commutative, $\Omega$ denotes the spectral space of $\mathcal{A}$ which is locally compact in the weak * topology and $C_{0}^{\infty}(\Omega)$ denotes the set of all continuous functions on $\Omega$ vanishing at infinity. For other notations, we will follow $[9$.

For the commutativity of $C^{*}$-algebras, a lot of results have been obtained from various points of view, for example, the nilpotent and ideal characterizations given by I. Kaplansky (see [2] and [7), the numerical characterizations given by M. J. Crabb, J. Duncan and C. M. McGregor (see [1]) and J. Duncan and P. J. Taylor (see 4]), the order characterizations given by T. Ogasawara (see [11), S. Sherman (see [12]), M. J. Crabb, J. Duncan and C. M. McGregor (see [1]) and M. Fukamiya, M. Misonou and Z. Takeda (see [5]), ${ }^{*}$-representation characterizations given by C. F. Skau (see [13]) and S. Wright (see [14]) and spectral characterizations given by R. Nakamoto (see [10]) and Y. Kato (see [8]).

From [6], we know that some continuous real-valued functions defined on a subset $S$ of the real field $\mathbf{R}$ are operator-monotonic increasing on $S$ and others are not. Therefore, we introduce the following concept.

Definition 1. Let $\mathcal{A}$ be a $C^{*}$-algebra and $f$ a continuous real-valued function defined on a subset $S$ of the real field $\mathbf{R}$. We say that the function $f$ is operatormonotonic increasing on $\mathcal{A}$ associated with $S$ if $f(x) \leq f(y)$ in $\mathcal{A}+\mathbf{C}$ whenever $x$ and $y$ are self-adjoint elements of $\mathcal{A}, x \leq y$ and $\sigma(x) \cup \sigma(y) \subseteq S$. We denote by

Received by the editors November 4, 1998 and, in revised form, June 4, 1999.

2000 Mathematics Subject Classification. Primary 46L05.

Key words and phrases. Commutativity for $C^{*}$-algebras, operator-monotonic increasing function, positive element. 
$M_{\mathcal{A}}(S)$ the set of operator-monotonic increasing functions on $\mathcal{A}$ associated with the subset $S$ of the real field $\mathbf{R}$.

The exponential function plays an important role in the study of $C^{*}$-algebras (see [3, [9], etc.). In this paper, we will give another order characterization of commutativity for $C^{*}$-algebras through operator-monotonic increasing functions.

\section{LEMMAS}

In this section, we derive the basic properties of operator-monotonic increasing functions on a $C^{*}$-algebra associated with a subset of the real field $\mathbf{R}$.

Lemma 1. Let $\mathcal{A}$ be a $C^{*}$-algebra and $S$ a subset of the real field $\mathbf{R}$.

(i) If $f \in M_{\mathcal{A}}(S)$, then $f$ is a monotonic increasing function on $S$.

(ii) $M_{\mathcal{A}}(S)$ is a convex cone.

(iii) If $f$ belongs to $M_{\mathcal{A}}(S)$ and takes values in some subset $T$ of the real field $\mathbf{R}$ and $g$ belongs to $M_{\mathcal{A}}(T)$, then the composition $(g \circ f)(t)=g(f(t))$ is also in $M_{\mathcal{A}}(S)$.

Proof. Direct verification according to Definition 1.

Corollary 1. Let $\mathcal{A}$ be a $C^{*}$-algebra. Then the function $f(t)=t^{2}+a t+b, a, b \in$ $\mathbf{R}$ and $t \in\left[-\frac{a}{2}, \infty\right)$, is operator-monotonic increasing on $\mathcal{A}$ if and only if $\mathcal{A}$ is commutative.

Proof. Let

$$
\begin{gathered}
g(t)=t^{2}, t \in[0, \infty), \\
h(t)=t+\frac{a}{2}, t \in\left[-\frac{a}{2}, \infty\right),
\end{gathered}
$$

and

$$
k(t)=t+b-\frac{a^{2}}{4}, t \in[0, \infty) .
$$

Then

$$
f(t)=(k \circ g \circ h)(t), t \in\left[-\frac{a}{2}, \infty\right),
$$

and by Lemma 1(ii), the functions $h$ and $k$ are operator-monotonic increasing on $\mathcal{A}$.

If $\mathcal{A}$ is a commutative $C^{*}$-algebra, then the function $g$ is operator-monotonic increasing on $\mathcal{A}$ by Definition 1. Therefore, the function $f$ is operator-monotonic increasing on $\mathcal{A}$ according to Lemma 1(iii).

Conversely, if the function $f$ is operator-monotonic increasing on $\mathcal{A}$, then, by Lemma 1(ii), the function $p(t)=\left(t+\frac{a}{2}\right)^{2}, t \in\left[-\frac{a}{2}, \infty\right)$, is operator-monotonic increasing on $\mathcal{A}$, and hence the function $q(t)=t^{2}, t \in[0, \infty)$, is operator-monotonic increasing on $\mathcal{A}$. It follows that $\mathcal{A}$ is a commutative $C^{*}$-algebra by T. Ogasawara's order characterization theorem (see [11]).

Lemma 2. Let $\mathcal{A}$ be a $C^{*}$-algebra and $f(t), f_{k}(t), k=1,2, \ldots$, continuous realvalued functions defined on a subset $S$ of the real field $\mathbf{R}$. If $f_{k} \in M_{\mathcal{A}}(S), k=$ $1,2, \ldots$, and $\left\{f_{k}\right\}$ converges to $f$ uniformly on compact subsets of $S$ when $k \rightarrow \infty$, then $f$ belongs to $M_{\mathcal{A}}(S)$. 
Proof. Take $x, y$ in $\mathcal{A}_{H}$ such that

$$
x \leq y
$$

and

$$
\sigma(x) \cup \sigma(y) \subseteq S .
$$

Let $\left\{e_{x}(\lambda)\right\}$ and $\left\{e_{y}(\lambda)\right\}$ be the spectral families of $x$ and $y$, respectively. By spectral theory, we have

$$
x=\int_{\sigma(x)} \lambda d e_{x}(\lambda)
$$

and

$$
y=\int_{\sigma(y)} \lambda d e_{y}(\lambda)
$$

Since $\left\{f_{k}\right\}$ converges to $f$ uniformly on compact subsets of $S$, we get

$$
\begin{aligned}
f(x) & =\int_{\sigma(x)} f(\lambda) d e_{x}(\lambda) \\
& =\lim _{k \rightarrow \infty} \int_{\sigma(x)} f_{k}(\lambda) d e_{x}(\lambda) \\
& =\lim _{k \rightarrow \infty} f_{k}(x)
\end{aligned}
$$

and

$$
\begin{aligned}
f(y) & =\int_{\sigma(y)} f(\lambda) d e_{y}(\lambda) \\
& =\lim _{k \rightarrow \infty} \int_{\sigma(y)} f_{k}(\lambda) d e_{y}(\lambda) \\
& =\lim _{k \rightarrow \infty} f_{k}(y)
\end{aligned}
$$

(see [7, Sec. 5.2]). The premise that $\left\{f_{k}: k=1,2, \ldots\right\} \subseteq M_{\mathcal{A}}(S)$ implies that

$$
f_{k}(x) \leq f_{k}(y), k=1,2, \ldots \text {. }
$$

Therefore, $f(x) \leq f(y)$, and hence $f \in M_{\mathcal{A}}(S)$.

\section{MAIN THEOREM AND COROLLARY}

Now we come to the main results.

Theorem 1. Let $\mathcal{A}$ be a $C^{*}$-algebra. Then the function $f(t)=e^{t}, t \in[0, \infty)$, is operator-monotonic increasing on $\mathcal{A}$ if and only if $\mathcal{A}$ is a commutative $C^{*}$-algebra.

Proof. Suppose $\mathcal{A}$ is a commutative $C^{*}$-algebra. Then $\mathcal{A}$ is isometrically * isomorphic to $C_{0}^{\infty}(\Omega)$ (see [9, p. 73]). Now it is clear that the function $f(t)=e^{t}, t \in[0, \infty)$, is operator-monotonic increasing on $\mathcal{A}$.

Conversely, assume that $x$ and $y$ are elements in $\mathcal{A}_{+}$and $x \leq y$. Letting $0<\epsilon<$ 1 , we have

$$
x+\epsilon \leq y+\epsilon .
$$


Because the function $t \longmapsto-t^{-1}, t \in(0, \infty)$, is operator-monotonic increasing on $\mathcal{A}$ (see [6]), the function

$$
g_{\alpha}(t)=\frac{1}{\alpha+1}-\frac{1}{\alpha+t}, t \in(0, \infty)
$$

where $\alpha \geq 0$, is operator-monotonic increasing on $\mathcal{A}$. For $c>0$, let

$$
B=\max \left\{\frac{1-\epsilon}{e^{c}-1}-\epsilon, 0\right\} .
$$

Then, when $b>B$, we have

$$
\left|\int_{b}^{\infty} g_{\alpha}(t) d \alpha\right| \leq \ln \frac{b+1}{b+\epsilon}<c
$$

for all $t \in[\epsilon,\|y\|+\epsilon]$. So $\int_{0}^{\infty} g_{\alpha}(t) d \alpha$ converges uniformly on $[\epsilon,\|y\|+\epsilon]$, and hence the function

$$
s(t)=\ln t-\int_{0}^{\infty} g_{\alpha}(t) d \alpha
$$

is continuous on $[\epsilon,\|y\|+\epsilon]$. Let $t_{0} \in[\epsilon,\|y\|+\epsilon]$ such that

$$
\left|\ln t_{0}-\int_{0}^{\infty} g_{\alpha}\left(t_{0}\right) d \alpha\right|=\max _{t \in[\epsilon,\|y\|+\epsilon]}\left\{\left|\ln t-\int_{0}^{\infty} g_{\alpha}(t) d \alpha\right|\right\} .
$$

Then for $\delta>0$, there are an integer $n$ and an equidistant division

$$
0=\alpha_{0}<\alpha_{1}<\ldots<\alpha_{m}=n
$$

of the interval $[0, n]$ such that

$$
\left|\ln t-\frac{n}{m} \sum_{k=1}^{m} g_{\alpha_{k}}(t)\right|<\delta
$$

for all $t \in[\epsilon,\|y\|+\epsilon]$. So we have

$$
\begin{aligned}
\ln (x+\epsilon) & \leq \frac{n}{m} \sum_{k=1}^{m} g_{\alpha_{k}}(x+\epsilon)+\delta \\
& \leq \frac{n}{m} \sum_{k=1}^{m} g_{\alpha_{k}}(y+\epsilon)+\delta \\
& \leq \ln (y+\epsilon)+2 \delta
\end{aligned}
$$

which shows that $\ln (x+\epsilon) \leq \ln (y+\epsilon)$. Choose real number $K$ such that $K \geq$ $-2 \ln (t+\epsilon), t \in[0, \infty)$. Since the function $f(t)=e^{t}, t \in[0, \infty)$, is operatormonotonic increasing on $\mathcal{A}$, we get

$$
e^{2 \ln (x+\epsilon)+K} \leq e^{2 \ln (y+\epsilon)+K},
$$

and hence

$$
(x+\epsilon)^{2} \leq(y+\epsilon)^{2}
$$

(see [7] Sec. 3.3]). Therefore, for $\epsilon \in(0,1)$, the functions $f_{\epsilon}(t)=t^{2}+2 \epsilon t+\epsilon^{2}, t \in$ $[0, \infty)$, are operator-monotonic increasing on $\mathcal{A}$. It is clear that $\left\{g_{n}\right\}=\left\{f_{\frac{1}{n}}\right\}$ converges to $g(t)=t^{2}, t \in[0, \infty)$, uniformly on compact subsets of $[0, \infty)$. By Lemma 2, the function $f(t)=t^{2}, t \in[0, \infty)$, is operator-monotonic increasing on 
$\mathcal{A}$. So $\mathcal{A}$ is a commutative $C^{*}$-algebra by T. Ogasawara's order characterization theorem.

Corollary 2. Let $\mathcal{A}$ be a $C^{*}$-algebra. Then $\mathcal{A}$ is commutative if and only if $e^{x+y}=$ $e^{x} e^{y}$ in $\mathcal{A} \dot{+} \mathbf{C}$ for all $x, y \in \mathcal{A}_{+}$.

Proof. If $\mathcal{A}$ is a commutative $C^{*}$-algebra, it is clear that $e^{x+y}=e^{x} e^{y}$ in $\mathcal{A} \dot{+} \mathbf{C}$ for all $x, y \in \mathcal{A}_{+}$.

Conversely, assume that $x, y \in \mathcal{A}_{+}$and $x \leq y$. Let $\mathcal{B}$ be the $C^{*}$-subalgebra generated by $(y-x)$. Then $\mathcal{B}$ is isometrically ${ }^{*}$ isomorphic to $C_{0}^{\infty}(\sigma(y-x) \backslash\{0\})$ (see [9. p. 73]). Because $(y-x)(t) \geq 0, t \in \sigma(y-x) \backslash\{0\}, e^{y-x}(t) \geq 1$, that is, $e^{y-x} \geq 1$. So

$$
e^{x}=e^{\frac{x}{2}} \cdot 1 \cdot e^{\frac{x}{2}} \leq e^{\frac{x}{2}} \cdot e^{y-x} \cdot e^{\frac{x}{2}}=e^{y} .
$$

Thus the function $f(t)=e^{t}, t \in[0, \infty)$, is operator-monotonic increasing on $\mathcal{A}$. By Theorem $1, \mathcal{A}$ is a commutative $C^{*}$-algebra.

\section{ACKNOWLEDGMENT}

The author is grateful to Professor Bingren Li for his going over the manuscript of this paper and his suggestions. The author also expresses his thanks to referee for his or her suggestions.

\section{REFERENCES}

1. M. J. Crabb, J. Duncan and C. M. McGregor, Characterizations of commutativity for $C^{*}$ algebras, Glasgow Math. J. 15 (1974), 172-175. MR 50:14252

2. J. Dixmier, $C^{*}$-algebras, North-Holland Mathematical Library, Vol. 15, North-Holland Publishing Co., Amsterdam, 1977. MR 56:16388

3. R. S. Doran and V. A. Belfi, Characterizations of $C^{*}$-algebras, The Gelfand-Naimark Theorems, Marcel Dekker, Inc., New York, 1986. MR 87k:46115

4. J. Duncan and P. J. Taylor, Norm Inequalities for $C^{*}$-algebras, Proc. Roy. Soc. Edinburgh, Sect. A, 75 (1975/76), 119-129. MR 56:12896

5. M. Fukamiya, M. Misonou and Z. Takeda, On order and commutativity of $B^{*}$-algebras, Tôhoku Math. J. (2)6 (1954), 89-93. MR 16:376

6. F. Hansen and G. K. Pedersen, Jensen's inequality for operators and Lowner's theorem, Math. Ann. 258 (1982), 229-241. MR 83g:47020

7. R. V. Kadison and J. R. Ringrose, Foundamentals of the theory operator algebras, Vol. 1, Elementary theory, Pure and Applied Mathematics, 100, Academic Press, Inc., New YorkLondon, 1983. MR 85j:46099

8. Y. Kato, A characterization of commutative $C^{*}$-algebras by normal approximate spectra, Math. Japon. 24 (1979/80), 209-210. MR 81f:46071

9. B. R. Li, Introduction to operator algebras, World Scientific, Singapore, 1992. MR 94b:46083

10. R. Nakamoto, A spectral characterization of commutative $C^{*}$-algebras, Math. Japon. 24 (1979/80), 399-400. MR 81h:46074

11. T. Ogasawara, A theorem on operator algebras, J. Sci. Hiroshima Univ. Ser. A 18 (1955), 307-309. MR 17:514

12. S. Sherman, Order in operator algebras, Amer. J. Math. 73 (1951), 227-232. MR 13:47

13. C. F. Skau, Orthogonal measures on the state space of a $C^{*}$-algebra, in: Algebras in Analysis (Conference Proceedings, Academic Press, London, 1975), 272-303. MR 54:8302

14. S. Wright, On factor states, Rocky Mountain J. Math. 12 (1982), 569-579. MR 83j:46075

Institute of Mathematics, Academia Sinica, Beijing 100080, China

E-mail address: wwu@math03.math.ac.cn

Current address: Department of Mathematics, East China Normal University, Shanghai 200062, China

E-mail address: wwu@math.ecnu.edu.cn 